\title{
Isothermal Self-Assembly of Complex DNA Structures under Diverse and Biocompatible Conditions
}

\author{
Cameron Myhrvold, ${ }^{\dagger, \ddagger}$ Mingjie Dai, ${ }^{\ddagger}{ }^{\ddagger}$ Pamela A. Silver, ${ }^{\dagger, \ddagger}$ and Peng Yin ${ }^{* \dagger, \ddagger}$
}

${ }^{\dagger}$ Department of Systems Biology, Harvard Medical School, Boston, Massachusetts 02115, United States

${ }^{\ddagger}$ Wyss Institute for Biologically Inspired Engineering and ${ }^{\S}$ Program in Biophysics, Harvard University, Boston, Massachusetts 02115, United States

\section{Supporting Information}

ABSTRACT: Nucleic acid nanotechnology has enabled researchers to construct a wide range of multidimensional structures in vitro. Until recently, most DNA-based structures were assembled by thermal annealing using high magnesium concentrations and nonphysiological environments. Here, we describe a DNA selfassembly system that can be tuned to form a complex target structure isothermally at any prescribed temperature or homogeneous condition within a wide range. We were able to achieve isothermal assembly between 15 and $69^{\circ} \mathrm{C}$ in a predictable fashion by altering the strength of strand-strand interactions in several different ways, for example, domain length, GC content, and linker regions between domains. We also observed the assembly of certain structures under biocompatible conditions, that is, at physiological $\mathrm{pH}$, temperature, and salinity in the presence of the molecular crowding agent polyethylene glycol (PEG) mimicking the cellular environment. This represents an important step toward the self-assembly of geometrically precise DNA or RNA structures in vivo.

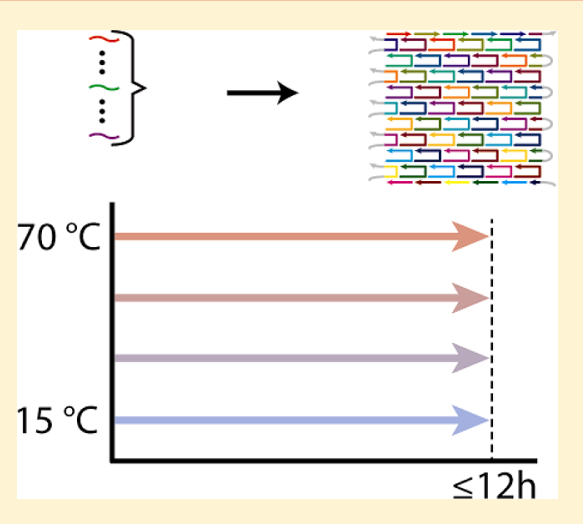

KEYWORDS: DNA nanotechnology, isothermal assembly, single-stranded tiles, biocompatible assembly, molecular crowding

$\mathrm{N}$ ucleic acid self-assembly has proven to be a powerful tool for constructing nanoscale structures due to the precise and predictable relationship between structure geometry and the encoded sequence. Over the past few decades, a wide range of DNA and RNA-based nanostructures have been demonstrated, including a variety of two-dimensional (2D) and threedimensional (3D) geometries ${ }^{1-14}$ and dynamic devices. ${ }^{15-22}$ Additionally, such nanostructures can interface with and organize molecules, cells and other materials, ${ }^{1,12,23-27}$ thereby enabling biophysical ${ }^{24,28}$ and potential biomedical ${ }^{23,29,30}$ applications. The self-assembly of individual strands into DNA nanostructures typically involves a thermal gradient in which the system temperature is first raised (e.g., to $80-90^{\circ} \mathrm{C}$ ) and then gradually lowered to room temperature. This thermal gradient can be replaced with a chemical gradient of a denaturing agent to isothermally assemble precisely controlled DNA origami structures. ${ }^{31}$ In addition to gradient-based assembly, researchers have demonstrated the isothermal formation of extended crystals ${ }^{12,32,33}$ using DNA and RNA tiles and discrete structures using DNA origami, ${ }^{34}$ both under homogeneous conditions.

Isothermal assembly of DNA tiles and origami under homogeneous conditions represents important progress for the assembly of complex DNA nanostructures. Compared with thermal annealing, assembling structures in homogeneous conditions leads to rapid structure formation with higher yield and quality. ${ }^{34}$ However, previous work on DNA tile crystals and origami still requires a highly optimized set of assembly conditions (temperature, salinity, etc.) that are structure-dependent and restricted to a narrow range, thereby limiting the scope of potential applications. In contrast, assembly across a wide range of conditions will increase the range of applications of DNA nanotechnology, especially when the assembly conditions are specified by the application, such as in vivo scaffolding ${ }^{12}$ of metabolic enzymes (where the assembly is required to happen under intracellular conditions) or in situ imaging $^{35}$ (where the assembly is required to happen under harsh denaturing conditions). Thus, the next important challenge is to devise a general method in which, given a particular homogeneous condition and a target shape, the user can design a system that assembles under that particular condition into the desired shape, e.g. the isothermal assembly of a particular shape under any prescribed temperature over a wide temperature range. Such capability will greatly expand the application scope where an assembly system can be designed to operate under diverse homogeneous conditions specified by the desired application. Here we provide a general solution to this challenge.

The key innovation in our approach is that we exploit the tunable nature of single-stranded tile (SST) structures and demonstrate structural designs that can assemble isothermally at a wide range of temperature and salt concentrations,

Received: May 28, 2013

Revised: July 4, 2013

Published: August 26, 2013 
including conditions mimicking intracellular environments. Compared with DNA origami, which typically uses a fixed scaffold sequence, single-stranded tiles (SSTs) give us the freedom to independently vary the sequence of any tile in the structure. We use this freedom to program the strength of strand-strand interactions, thereby shifting the optimal assembly temperature to a user-specified temperature. In other words, the assembly temperature of a structure is now an explicit design parameter. This will allow one to tune the design of a structure to fit a particular application.

We report the successful isothermal assembly of modified SST structures across a wide range of temperatures. Building upon our previous SST work, $5,13,14,36$ we test 11 compact 2D SST structures (Figure 1a) and 17 flexible variants with single-

a

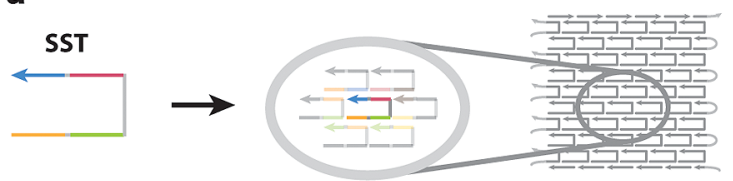

b

SST with linker

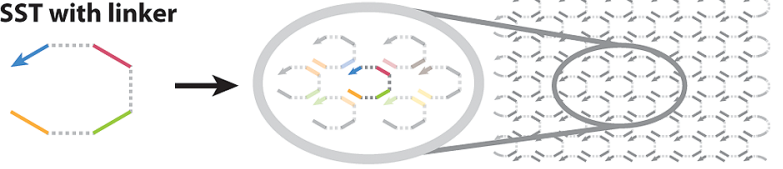

C

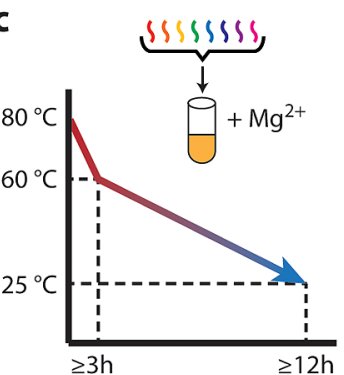

d

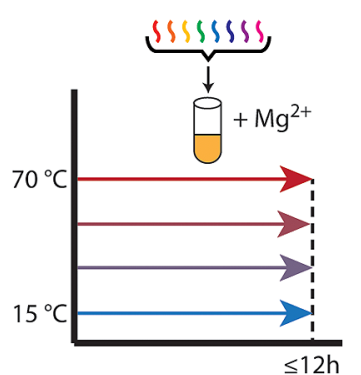

Figure 1. Self-assembly of DNA tiles under diverse and biocompatible conditions. (a,b) We use standard single-stranded tiles (SST; a) and SST tiles containing single-stranded linker regions between domains (b) to assemble $2 \mathrm{D}$ rectangular structures. A canonical SST has four domains that bind to four of its nearest neighbors. Each colored segment depicts an individual domain (unique in sequence and between 8 and 21 nt long in our study), a gray solid connection delineates the boundary between two domains, and a gray dotted line depicts a single-stranded linker region (between 1 and $16 \mathrm{nt}$ in our study). Each complete structure (shown on the right) consists of 66 unique SSTs with edges protected by polyT segments to avoid aggregation (light gray segments). (c) A schematic depicting the traditional thermal annealing protocol for structure assembly, where temperature is generally ramped above $60{ }^{\circ} \mathrm{C}$ and slowly decreased over at least $12 \mathrm{~h}$. (d) A schematic depicting our isothermal assembly protocol across a wide range of fixed temperatures and conditions for up to $12 \mathrm{~h}$.

stranded polyT linkers between the double-stranded domains (Figure 1b). ${ }^{36}$ Instead of the traditional thermal annealing protocol (Figure 1c), all structures were assembled isothermally (Figure 1d). Our approach therefore represents a general method for isothermally assembling complex nanostructures across a wide range of specified temperatures. We generally find higher assembly temperatures for structures with longer domains, and lower temperatures for those with linkers. We also show that the assembly temperature can be modulated by changing the strand-strand binding energy in several ways, such as altering GC content of binding domains or disrupting continuity of complementary segments.

Finally, we report successful assembly of designed DNA nanostructures under biocompatible conditions, that is, at physiological temperature and $\mathrm{pH}$, low salinity, and in the presence of molecular crowding agents. Flexible structures, which assemble at a wider range of conditions than their nonflexible counterparts, assemble well under biocompatible conditions. This represents a significant step toward the in vivo assembly of geometrically precise, nucleic acid nanostructures. More generally, this approach may enable additional biological applications of nanostructures in cases where in situ delivery of preassembled structures is not an option.

In our single-stranded tile (SST) system, each strand consists of four binding domains, with or without a single-stranded linker region between consecutive domains. In the presence of divalent cations, each strand is bound to its four neighbors via complementary domains, resulting in a self-assembled 2D SST structure. ${ }^{13}$ Most of our structures are derived from motifs in our recent work $^{36}$ and consist of 66 SSTs that form a 2D rectangular shape. Two categories of motifs are used. The motifs without linkers form $2 \mathrm{D}$ rectangles composed of 10 parallel helices (with length ranging from 108 to $252 \mathrm{nt}$ ) connected by periodic single-stranded crossovers. Motifs with polyT linkers form flexible rectangular, fish-net patterns containing short segments of DNA helices connected by single-stranded linkers at all junctions. We varied domain length, linker length, and other design parameters. For an overview of the system, see Supporting Information Figure S1 and Text S1.

SST structures can isothermally assemble across a wide range of temperatures (Figure 2). We tested a set of U-shaped motifs $^{13,36}$ to form $2 \mathrm{D}$ rectangular structures. By altering the domain lengths of the SST motifs from 8 to $21 \mathrm{nt}$, we were able to achieve isothermal assembly across a $54{ }^{\circ} \mathrm{C}$ range (from 15 to $69{ }^{\circ} \mathrm{C}$ ). Structure formation was assayed by gel electrophoresis and atomic force microscopy (AFM). A dominant product band was observed after isothermal assembly for $12 \mathrm{~h}$ at the indicated temperatures in the presence of $10 \mathrm{mM} \mathrm{Mg}{ }^{2+}$. The formation ranges indicated on the temperature line are a subset of the temperature ranges over which a dominant product band was observed on the gel (see Supporting Information Figure S2 for full range and raw gel data). In some cases, we observed the presence of high molecular weight bands or smears, presumably due to structure aggregation.

Imaging of the unpurified reaction or of the gel-purified product (indicated with an asterisk) revealed the formation of structures with the designed shapes. Structures with polyT linkers between domains were imaged after the addition of complementary polyA strands. They exhibited a wide range of conformations when imaged due to the flexibility inherent in the linker regions.

The assembly yield was quantified using native gel electrophoresis (Figure 3a). We assembled structures isothermally for $1 \mathrm{~h}$ (domain length $<16 \mathrm{nt}$ ) or $12 \mathrm{~h}$ (domain length $\geq 16 \mathrm{nt}$ ) at a range of temperatures and then quantified the gel yield in each lane (which corresponds to a particular, fixed assembly temperature) using the TotalLab Quant gel quantification software (Figure $3 \mathrm{~b}$ ). Here, the yield is defined as the ratio of the intensity of the product band divided by the intensity of the entire lane after proper background correction. 


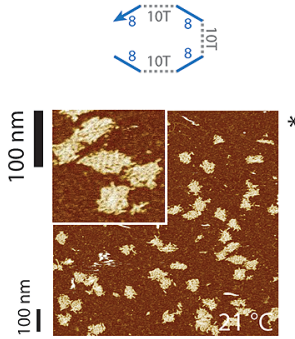

15182124
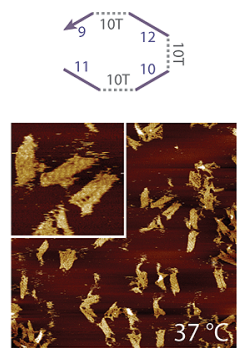

33353841
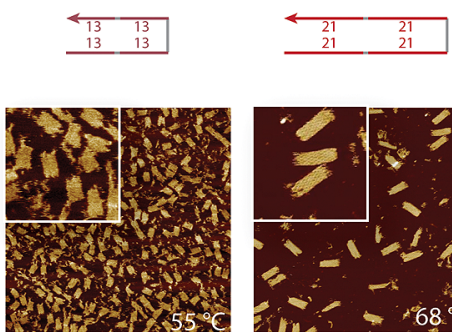

525557

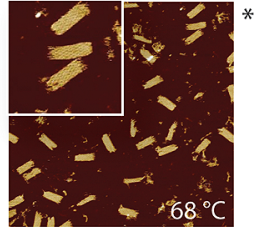

656769

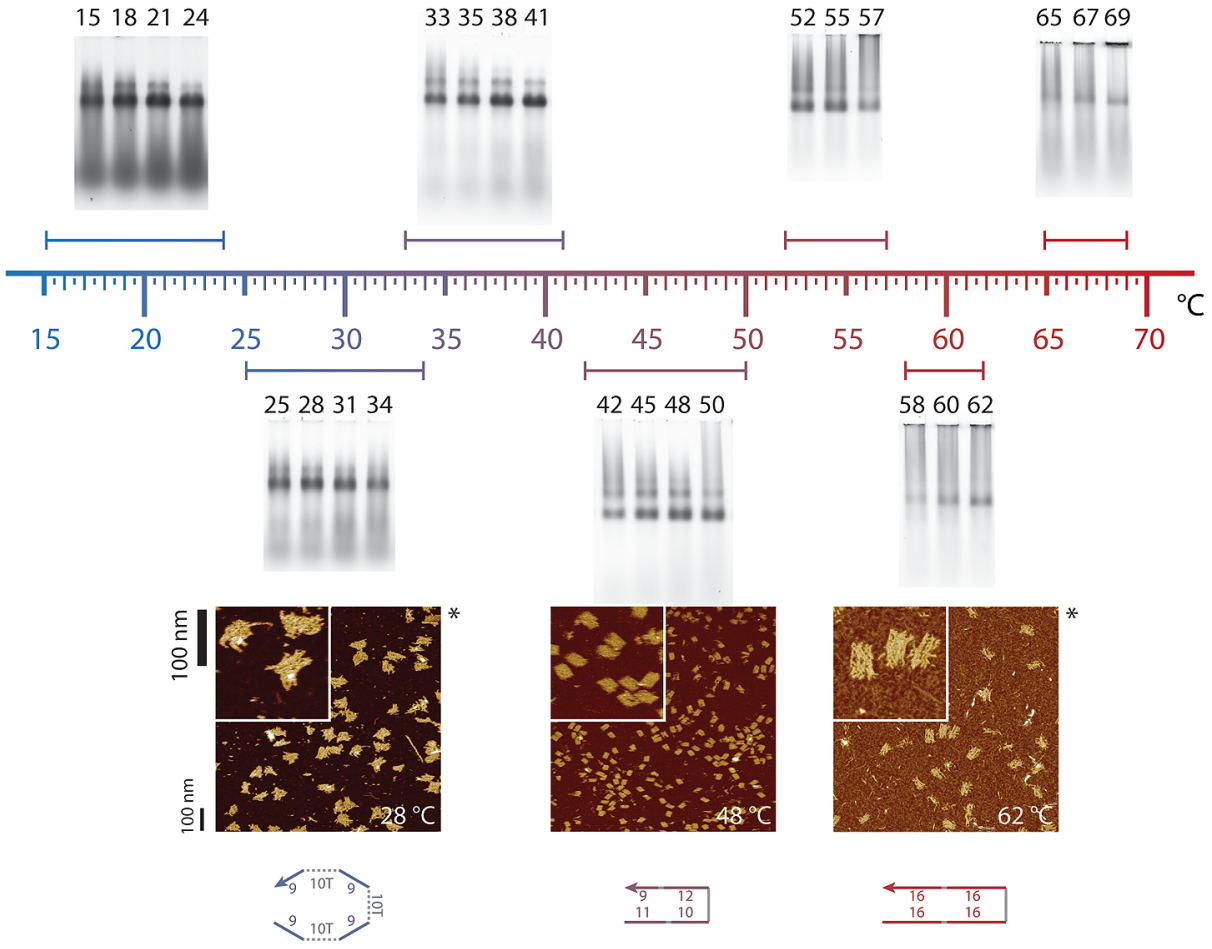

Figure 2. SST-based DNA nanostructures can assemble isothermally across a wide range of temperatures. Representative structures that assemble isothermally at different temperatures are shown and collectively cover the entire temperature range from $15{ }^{\circ} \mathrm{C}$ (blue) to $69{ }^{\circ} \mathrm{C}$ (red). Structures are ordered and displayed close to their optimal formation temperatures. For each structure, strand diagrams (outside), AFM images (middle), and gel electrophoresis results (inside) are shown. A tagged bar indicates part of the range of successful assembly temperatures shown by agarose gel electrophoresis (see Supporting Information Figure S2 for full gel images).The leftmost three structures contain 10T linkers between domains; the other structures do not, as shown in the strand diagrams. Numbers in strand diagrams indicate domain lengths (unit: nt). AFM images show the typical morphology at the indicated temperatures; images with an asterisk contain gel-purified structures. Structures were assembled isothermally for $12 \mathrm{~h}$ using $200 \mathrm{nM}$ of each strand and $10 \mathrm{mM} \mathrm{Mg}^{2+}$.

a

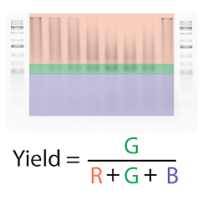

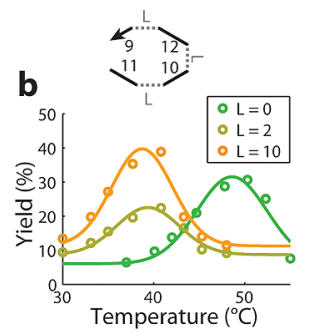
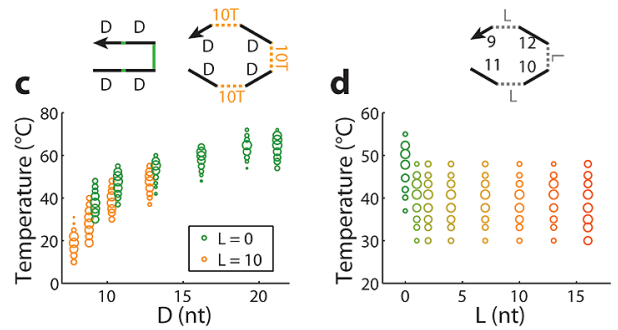

Figure 3. Quantification of optimal assembly temperature and effect of domain and linker lengths. Structures were assembled isothermally for $1 \mathrm{~h}$ across a temperature range using $200 \mathrm{nM}$ of each strand and $10 \mathrm{mM} \mathrm{Mg}{ }^{2+}$ and assayed using gel electrophoresis. (a) Gel images were quantified using TotalLab Quant software. Gel yield was defined as the integrated intensity of the formation band (green) divided by the total intensity of the lane (red + green + blue). (b) Gel yield of three representative structures $(L=0,2,10)$ and Gaussian fits (solid lines) are shown. (c,d) Formation yields as a function of temperature are shown for structures with varying domain lengths $(D=8-21 ; \mathrm{c})$ and linker lengths $(L=0-16$; d). Each circle represents a single yield quantification, with its radius proportional to the measured yield; colors indicate linker length from no linker $(L=0$; green) to $16 \mathrm{~T}$ linker $(L=16$; dark orange).

This is only an approximation of the true formation yield ${ }^{13}$ but is useful for comparing relative yields for assembly under varying conditions. The yield curves were then fit to a Gaussian function with a constant background using custom MATLAB software (Figure $3 \mathrm{~b}$ ). The yield as a function of temperature is 


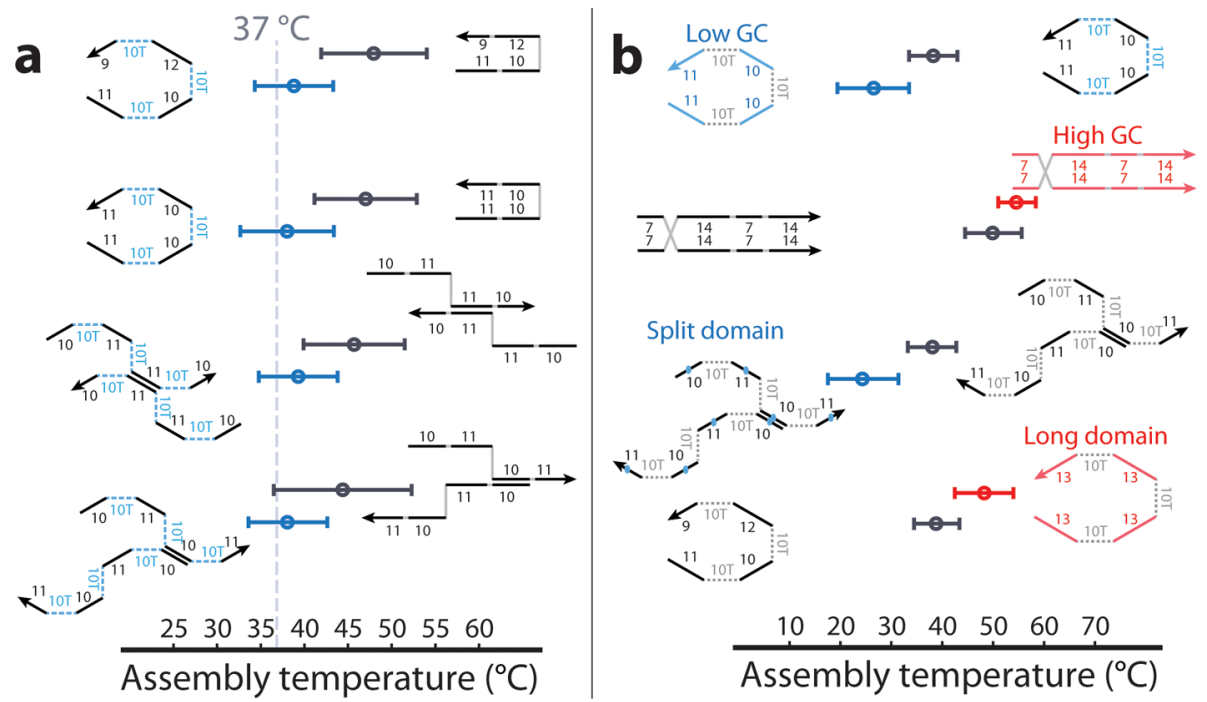

Figure 4. Optimal assembly temperatures can be rationally designed with linker domains and other methods. (a) Shifting to lower optimal assembly temperatures (blue) by addition of linkers (dashed line: physiological temperature). Strand diagrams of four representative designs with distinct strand geometry are shown. (b) Rational design of optimal assembly temperature (blue, lower; red, higher) with three other methods: altering the GC content of domains to 30\% (low GC) or 70\% (high GC), splitting domains in half and changing domain lengths. In the case of splitting domains, a single $\mathrm{T}$ nucleotide is inserted in the middle of each domain (blue dots) to split each strand into a 5-T-5-10T-6-T-5-10T-5-T-5-10T-5-T6 sequence pattern. In both panels, numbers in strand diagrams indicate domain lengths (unit: nt), tagged bars delimit the full width at halfmaximum (fwhm) of a Gaussian fit to the yield as a function of assembly temperature, and a circle denotes the optimal temperature (see Supporting Information Figure S4 for raw gel data).
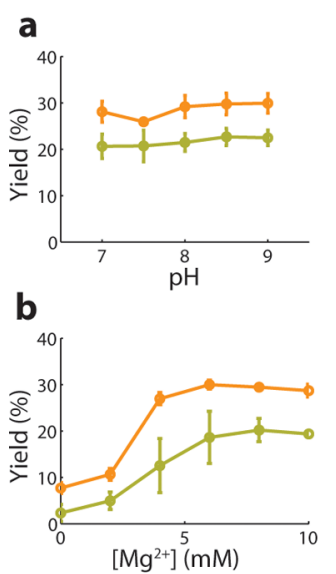

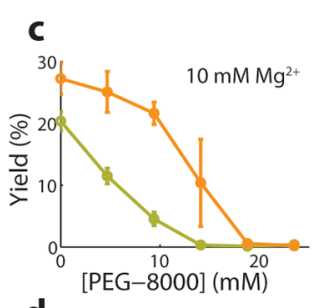

d

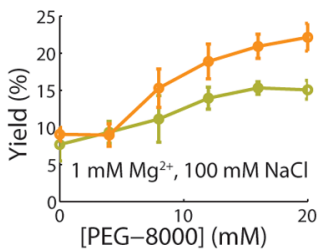

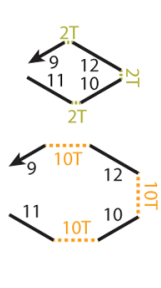

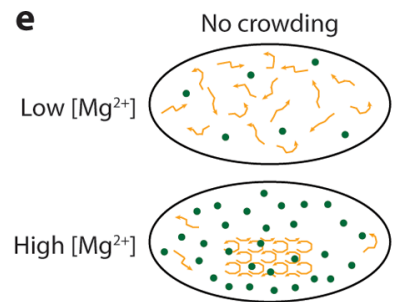

f $\left[\mathrm{Mg}^{2+}\right] \mathrm{L} \mathrm{H} \mathrm{H} \mathrm{L}$

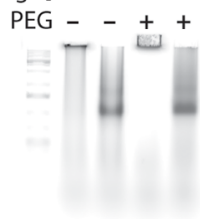

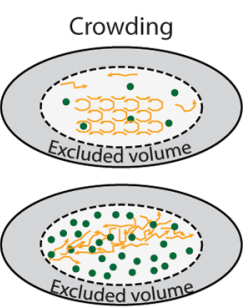

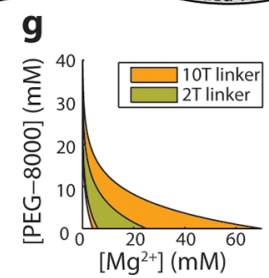

Figure 5. Assembly of structures under biocompatible conditions. (a-d) Effects of assembly conditions on formation yield of two representative structures (olive, $2 \mathrm{~T}$ linker; orange, 10T linker; see strand diagrams). Structures were assembled for $1 \mathrm{~h}$ at $37^{\circ} \mathrm{C}$ using $200 \mathrm{nM}$ of each strand. Yields are calculated as in Figure 3a. Error bars indicate the standard deviation based on $\geq 3$ replicate experiments. (a-c) Effects of $\mathrm{pH}$, $\left[\mathrm{Mg}^{2+}\right]$, and $[\mathrm{PEG}-$ 8000 ] on formation yield. Each parameter is varied alone while keeping the others at default values $\left(0.5 \times \mathrm{TE}\right.$ buffer, $\mathrm{pH} 8,10 \mathrm{mM} \mathrm{Mg}{ }^{2+}$, no PEG8000). HEPES buffer was used for the $\mathrm{pH} 7$ condition. (d) Combined effect of low salinity ( $\left.1 \mathrm{mM} \mathrm{Mg} \mathrm{Mg}^{2+}, 100 \mathrm{mM} \mathrm{NaCl}\right)$ and [PEG-8000]. (e) A schematic of the crowding model: formation takes place at in vitro (no crowding, high salinity) or biocompatible (crowding, low salinity) conditions. Orange: component strands. Dark green: magnesium ions. (f) Gel result for the 10T linker structure at the four conditions shown in (e): with or without PEG-8000 (-, $0 \mathrm{mM} ;+, 20 \mathrm{mM})$, with low and high salinity (L, $\left.1 \mathrm{mM} \mathrm{Mg}^{2+} 100 \mathrm{mM} \mathrm{Na}^{+} ; \mathrm{H}, 10 \mathrm{mM} \mathrm{Mg}^{2+}\right)$. (g) A simple mathematical model of crowding, based on effective concentrations (see Supporting Information Text $\mathrm{S} 3$ for details), predicts assembly under low $\left[\mathrm{Mg}^{2+}\right]$ and high [PEG-8000] conditions.

well-fit by a Gaussian function, indicating that our structures assemble optimally at a specific isothermal temperature.

We show dependence between the assembly temperature and the domain length (Figure 3c; see Supporting Information Figure S3a for raw gel data). The Gaussian fits obtained from curve fitting to the gel quantification data allowed us to obtain a mean and a full-width at half-maximum (fwhm) for each structure that define an optimal temperature and an optimal temperature range, respectively. The formation range is indicated by the extent of the scatterplot, and the relative yields at each temperature are indicated by the diameter of the marker. We examined two sets of structures, those with no linker between domains (green) and those with a 10T linker between domains (orange). In both cases, we observed that the formation range was a monotonically increasing function of the domain length with diminishing returns at higher temperatures. It should be noted that since all of our domains have a similar GC content (around 50\%) that domain length is a proxy for the strength of strand-strand interactions in these experiments. 
Linkers between domains caused an $\sim 10{ }^{\circ} \mathrm{C}$ downward shift in the assembly temperature (Figure $3 \mathrm{~d}$; see Supporting Information Figure S3b for raw gel data). Even a single $\mathrm{T}$ nucleotide between domains was sufficient to shift the assembly temperature; additional $\mathrm{T}$ nucleotides did not contribute to the temperature shift. As in Figure $3 \mathrm{~b}$, we show the formation range using a scatterplot, where the relative yield at each temperature is indicated by the diameter of the marker. The effect of the linkers did not depend on the polyT sequence; a linker consisting of 10 A nucleotides caused a similar downward shift in assembly temperature (see Supporting Information Figure S3c).

We redesigned many structures to assemble at the physiological temperature using polyT linkers (Figure 4a; see Supporting Information Figure S4a for raw gel data). As shown, the effect of polyT linkers was similar for several different strand geometries, causing a downward shift of the assembly temperature of $7-10{ }^{\circ} \mathrm{C}$. In all cases, the redesigned structures formed well at the physiological temperature $\left(37^{\circ} \mathrm{C}\right)$, indicating the generality of this approach for programming the assembly temperature of DNA nanostructures. Furthermore, we demonstrated several additional strategies to shift the assembly temperature (Figure $4 \mathrm{~b}$; see Supporting Information Figure S4b for raw gel data): altering the strength of strandstrand interactions via the GC content, changing the length of the complementary domains within a structure, or splitting domains in half by inserting a single $\mathrm{T}$ nucleotide. Thus, there exists a wide variety of ways to program the assembly temperature of a DNA nanostructure. This should enable structures with varying geometries and morphologies that assemble at desired temperatures.

Flexible SST DNA structures assembled under biocompatible conditions. A number of structure designs assembled well at the physiological temperature $\left(37^{\circ} \mathrm{C}\right.$; Figures 2 , 3, and 4). However, there exist other environmental variables, such as $\mathrm{pH}$, salinity, and molecular crowding, which must be accounted for in order for structure assembly to be biocompatible. We tested two structure designs which assembled well at $37^{\circ} \mathrm{C}$ in 1 $\mathrm{h}$ (Figure $3 \mathrm{~b}$ ), one with a $2 \mathrm{~T}$ linker (olive) and one with a $10 \mathrm{~T}$ linker (orange) by systematically varying other environmental variables in vitro (Figure 5; see Supporting Information Figure S5 for raw gel data). Yields are determined as in Figure 3a.

Physiological $\mathrm{pH}$ is compatible with structure assembly (Figure 5a). We varied the $\mathrm{pH}$ of the buffer in which the structures were assembled from 7 to 9 , which encompassed the physiological $\mathrm{pH}$ range. Both structures assembled well across this $\mathrm{pH}$ range, indicating that the physiological $\mathrm{pH}$ should not prevent SST structures from self-assembling.

Low salinity hindered structure formation (Figure 5b). DNA nanostructures are typically assembled in vitro with a $\mathrm{Mg}^{2+}$ concentration in excess of $10 \mathrm{mM}^{4,6,13,37}$ Recently, it was shown that sodium ions can also be interchanged for magnesium ions in some cases. ${ }^{38}$ However, the free concentration of magnesium equivalents in the $E$. coli cytoplasm is approximately $2-4 \mathrm{mM}\left(1-2 \mathrm{mM} \mathrm{Mg}^{2+}, 180-\right.$ $200 \mathrm{mM} \mathrm{Na}^{+}$or $\mathrm{K}^{+}{ }^{39,40}$ ). Our flexible SST designs assembled close to optimally at as low as $6 \mathrm{mM} \mathrm{Mg}^{2+}$ (2T linker, olive) or $4 \mathrm{mM} \mathrm{Mg}^{2+}$ (10T linker, orange), approaching but not quite reaching the biologically relevant salinity.

Crowded conditions decreased the assembly yield (Figure 5c). A crowded environment was simulated using polyethylene glycol with an average molecular weight of 8000 Da (PEG8000 ). To test the effect of crowding on structure assembly, the
PEG-8000 concentration was varied from 0 to $23.5 \mathrm{mM}$. A $\mathrm{Mg}^{2+}$ concentration of $10 \mathrm{mM}$ was used in these experiments. We observed a decline in yield for both structure designs with increasing PEG-8000 concentrations, combined with the appearance of a high molecular weight band indicative of aggregation (see Supporting Information Figure S5c for raw gel data).These results indicate that a combination of crowding and high magnesium decreases assembly yield. Additionally, we have noted that the long linker design (orange) had a consistently higher yield than the short linker design (olive) under the conditions we tested (Figure 5a-d), perhaps due to increased flexibility enhancing the assembly process (e.g., by relieving the electrostatic repulsion between parallel DNA helices).

Molecular crowding compensated for low magnesium, thereby enhancing structure assembly (Figure 5d). When considered separately, both salinity and crowding pose challenges to biocompatible assembly of tile-based DNA nanostructures. However, when we tested the two conditions together by varying the PEG- 8000 concentration from 0 to 20 $\mathrm{mM}$ with low salinity $\left(1 \mathrm{mM} \mathrm{Mg}{ }^{2+}, 100 \mathrm{mM} \mathrm{Na}{ }^{+}\right)$at $\mathrm{pH} 7.5$, we observed that increased crowding actually improved the assembly yield. This effect was seen for both short (olive) and long linker designs (orange). Together, these results indicate that SST DNA nanostructures can be designed to assemble under biocompatible conditions.

We conjecture that molecular crowding enhances structure assembly by increasing the effective magnesium concentration (Figure 5e). In the absence of crowding, high salinity is required for assembly (top left), but in the presence of crowding high salinity inhibits assembly. This model is consistent with our experimental results; structure assembly (product band) is observed under high salinity with no crowding and low salinity with crowding but not under low salinity with no crowding or under high salinity with crowding (Figure 5f). We used this insight to construct a quantitative model of the effect of molecular crowding on structure assembly and predict a range of conditions under which assembly is likely to occur, indicated by the shaded regions (Figure 5g; see Supporting Information Text S3 and Figure S6 for details).

SST structures can be tuned to assemble isothermally across a range of temperatures from 15 to $69{ }^{\circ} \mathrm{C}$. Additionally, we demonstrated four different ways to modulate the assembly temperature of a structure: changing domain length, altering domain GC content, adding a linker between domains, or splitting a domain in half. This has enabled us to design SST structures that can assemble well under biocompatible conditions. More generally, we now have the capability to design structures that can assemble under a diverse range of specified conditions. We noticed that structures tend to assemble with lower yield at extreme temperatures or conditions. However, the yield at a particular condition, or the range of assembly conditions, can be increased by extending the reaction time. Together, these results indicate that the isothermal SST assembly process is remarkably robust to the assembly temperature and conditions.

Our ability to assemble structures at room temperature can enable the study of structure assembly in real time using atomic force microscopy ${ }^{22,32}$ or super-resolution microscopy techniques; $^{41}$ at the other end of the spectrum we can assemble structures at temperatures above the typical melting temperature of previous DNA nanostructures. More broadly, our 
unique ability to control the assembly temperature of DNA nanostructures allows structured to be tailored to in vivo or in situ applications where control of the ambient temperature may not be feasible. Our success in biocompatible assembly is particularly exciting because it is a key step toward in vivo assembly of geometrically precise nanostructures from many individual DNA or RNA components. Furthermore, biocompatible assembly takes us beyond the paradigm of annealing structures over many hours under precisely controlled reaction conditions. Our approach of rationally modulating the assembly conditions should be generalizable to other types of structures with a few potential constraints. One such constraint is that the component strands should not contain significant secondary structures that hinder their interactions with other strands. For example, the secondary structure of a typical M13 DNA origami scaffold strand may interfere with the designed structure formation at low temperatures. A scaffold with designed sequence could be used to overcome this constraint.

Beyond tunable SST structures, control over the assembly conditions could perhaps be further improved by exploiting toe-hold-mediated ${ }^{42}$ strand displacement cascades, ${ }^{17,43}$ for example, using the triggered isothermal assembly of reconfigurable hairpins. ${ }^{18,35,44}$ Although the resulting hairpin-based structures (e.g., branched junctions, ${ }^{18}$ polymers, ${ }^{35,44,45}$ or dendrimers ${ }^{18}$ ) are not geometrically precise and typically involve very few component strands, they can be formed dynamically via the introduction of an initiator strand. Additionally, a simple two-component toe-hold exchange based system has been shown be robust to temperature, salinity, and concentration. ${ }^{46}$ Thus, by integrating robustness of toe-hold based systems with the tunability and geometrical control of the SST systems described here, one may potentially design self-assembly systems that could allow for assembly across a wide range of temperatures and conditions without the need for condition-specific tuning.

A rational next step after biocompatible assembly is to assemble geometrically precise RNA nanostructures in vivo and to use them to organize metabolic enzymes or other functional biomolecules. $^{12}$ One could build upon the knowledge and experience from DNA SST assembly to design and rapidly prototype RNA structures that assemble under biocompatible conditions and act as scaffolds. With greater freedom in design geometry and positioning of protein-binding sites, one would be able to design and test interactions between multiple functional molecules across varying distances and geometrical patterns. One can thus survey a wider design space than has been achieved with previous protein ${ }^{47,48}$ or RNA $^{12}$ scaffolds. DNA or RNA nanostructures could be delivered or expressed as individual strands and assembled directly at the site of interest. Taken together, our results present a new opportunity to assemble precise, programmable structures in biological and cellular environments, which could lead to exciting in vitro and in vivo applications.

\section{ASSOCIATED CONTENT}

\section{S Supporting Information}

Supplementary methods, structure naming conventions, notes about experiments, modeling details, and six supplementary figures. This material is available free of charge via the Internet at http://pubs.acs.org.

\section{AUTHOR INFORMATION}

\section{Notes}

The authors declare no competing financial interest.

\section{ACKNOWLEDGMENTS}

We thank Bryan Wei, Dave Zhang, Erik Winfree, and Robert Barish for discussion. This work was funded by a DARPA Living Foundries grant HR0011-12-C-0061 to P.A.S. and P.Y., an Office of Naval Research (ONR) Young Investigator Program award N000141110914 to P.Y., an ONR grant N000141010827 to P.Y., an National Science Foundation (NSF) Faculty Early Career Development Award 1054898 and an NSF grant 1162459 to P.Y. C.M. acknowledges a graduate research fellowship from the Fannie and John Hertz Foundation. M.D. acknowledges an international student research fellowship from the Howard Hughes Medical Institute.

\section{REFERENCES}

(1) Yan, H.; Park, S. H.; Finkelstein, G.; Reif, J. H.; LaBean, T. H. Science 2003, 301, 1882-4.

(2) Chworos, A.; Severcan, I.; Koyfman, A. Y.; Weinkam, P.; Oroudjev, E.; Hansma, H. G.; Jaeger, L. Science 2004, 306, 2068-72.

(3) Rothemund, P. W. K.; Papadakis, N.; Winfree, E. PLoS Biol. 2004, 2, e424.

(4) Rothemund, P. W. K. Nature 2006, 440, 297-302.

(5) Yin, P.; Hariadi, R. F.; Sahu, S.; Choi, H. M. T.; Park, S. H.; Labean, T. H.; Reif, J. H. Science 2008, 321, 824-6.

(6) Douglas, S. M.; Dietz, H.; Liedl, T.; Högberg, B.; Graf, F.; Shih, W. M. Nature 2009, 459, 414-8.

(7) Dietz, H.; Douglas, S. M.; Shih, W. M. Science 2009, 325, 725-

(8) Zheng, J.; Birktoft, J. J.; Chen, Y.; Wang, T.; Sha, R.; Constantinou, P. E.; Ginell, S. L.; Mao, C.; Seeman, N. C. Nature 2009, 461, 74-7.

(9) Andersen, E. S.; Dong, M.; Nielsen, M. M.; Jahn, K.; Subramani, R.; Mamdouh, W.; Golas, M. M.; Sander, B.; Stark, H.; Oliveira, C. L. P.; Pedersen, J. S.; Birkedal, V.; Besenbacher, F.; Gothelf, K. V; Kjems, J. Nature 2009, 459, 73-6.

(10) Yang, H.; McLaughlin, C. K.; Aldaye, F. A.; Hamblin, G. D.; Rys, A. Z.; Rouiller, I.; Sleiman, H. F. Nature Chem. 2009, 1, 390-6.

(11) Han, D.; Pal, S.; Nangreave, J.; Deng, Z.; Liu, Y.; Yan, H. Science 2011, 332, 342-6.

(12) Delebecque, C. J.; Lindner, A. B.; Silver, P. A.; Aldaye, F. A. Science 2011, 333, 470-4.

(13) Wei, B.; Dai, M.; Yin, P. Nature 2012, 485, 623-6.

(14) Ke, Y.; Ong, L. L.; Shih, W. M.; Yin, P. Science 2012, 338, 11771183.

(15) Sherman, W. B.; Seeman, N. C. Nano Lett. 2004, 4, 1203-1207.

(16) Yin, P.; Yan, H.; Daniell, X. G.; Turberfield, A. J.; Reif, J. H. Angew. Chem., Int. Ed. 2004, 43, 4906-11.

(17) Zhang, D. Y.; Turberfield, A. J.; Yurke, B.; Winfree, E. Science 2007, 318, 1121-5.

(18) Yin, P.; Choi, H. M. T.; Calvert, C. R.; Pierce, N. a Nature 2008, $451,318-22$.

(19) Omabegho, T.; Sha, R.; Seeman, N. C. Science 2009, 324, 6771

(20) Lund, K.; Manzo, A. J.; Dabby, N.; Michelotti, N.; JohnsonBuck, A.; Nangreave, J.; Taylor, S.; Pei, R.; Stojanovic, M. N.; Walter, N. G.; Winfree, E.; Yan, H. Nature 2010, 465, 206-10.

(21) Qian, L.; Winfree, E. Science 2011, 332, 1196-201.

(22) Wickham, S. F. J.; Bath, J.; Katsuda, Y.; Endo, M.; Hidaka, K.; Sugiyama, H.; Turberfield, A. J. Nat. Nanotechnol. 2012, 7, 169-73.

(23) Douglas, S. M.; Bachelet, I.; Church, G. M. Science 2012, 335, $831-4$.

(24) Derr, N. D.; Goodman, B. S.; Jungmann, R.; Leschziner, A. E.; Shih, W. M.; Reck-Peterson, S. L. Science 2012, 338, 662-5. 
(25) Acuna, G. P.; Möller, F. M.; Holzmeister, P.; Beater, S.; Lalkens, B.; Tinnefeld, P. Science 2012, 338, 506-10.

(26) Lin, C.; Jungmann, R.; Leifer, A. M.; Li, C.; Levner, D.; Church, G. M.; Shih, W. M.; Yin, P. Nature Chem. 2012, 4, 832-9.

(27) Maune, H. T.; Han, S.-P.; Barish, R. D.; Bockrath, M.; Iii, W. A. G.; Rothemund, P. W. K.; Winfree, E. Nat. Nanotechnol. 2010, 5, 616.

(28) Douglas, S. M.; Chou, J. J.; Shih, W. M. Proc. Natl. Acad. Sci. U.S.A. 2007, 104, 6644-8.

(29) Lee, H.; Lytton-Jean, A. K. R.; Chen, Y.; Love, K. T.; Park, A. I.; Karagiannis, E. D.; Sehgal, A.; Querbes, W.; Zurenko, C. S.; Jayaraman, M.; Peng, C. G.; Charisse, K.; Borodovsky, A.; Manoharan, M.; Donahoe, J. S.; Truelove, J.; Nahrendorf, M.; Langer, R.; Anderson, D. G. Nat. Nanotechnol. 2012, 7, 389-93.

(30) Liu, X.; Xu, Y.; Yu, T.; Clifford, C.; Liu, Y.; Yan, H.; Chang, Y. Nano Lett. 2012, 12, 4254-9.

(31) Jungmann, R.; Liedl, T.; Sobey, T. L.; Shih, W.; Simmel, F. C. J. Am. Chem. Soc. 2008, 130, 10062-3.

(32) Evans, C. G.; Hariadi, R. F.; Winfree, E. J. Am. Chem. Soc. 2012, 134, 10485-92.

(33) Schulman, R.; Yurke, B.; Winfree, E. Proc. Natl. Acad. Sci. U.S.A.

2012, 109, 6405-10.

(34) Sobczak, J. J.; Martin, T. G.; Gerling, T.; Dietz, H. Science 2012, $338,1458-61$.

(35) Dirks, R. M.; Pierce, N. A. Proc. Natl. Acad. Sci. U.S.A. 2004, 101, 15275-8.

(36) Wei, B.; Dai, M.; Myhrvold, C.; Ke, Y.; Jungmann, R.; Yin, P. J. Am. Chem. Soc., in press.

(37) Winfree, E.; Liu, F.; Wenzler, L. a; Seeman, N. C. Nature 1998, 394, 539-44.

(38) Martin, T. G.; Dietz, H. Nat. Commun. 2012, 3, 1103.

(39) Alatossava, T.; Jütte, H.; Kuhn, A.; Kellenberger, E. J. Bacteriol. 1985, 162, 413-9.

(40) Shabala, L.; Bowman, J.; Brown, J.; Ross, T.; McMeekin, T.; Shabala, S. Environ. Microbiol. 2009, 11, 137-48.

(41) Jungmann, R.; Steinhauer, C.; Scheible, M.; Kuzyk, A.; Tinnefeld, P.; Simmel, F. C. Nano Lett. 2010, 10, 4756-61.

(42) Yurke, B.; Turberfield, A. J.; Mills, A. P., Jr.; Simmel, F. C.; Neumann, J. L. Nature 2000, 406, 605-608.

(43) Seelig, G.; Soloveichik, D.; Zhang, D. Y.; Winfree, E. Science (New York, N.Y.) 2006, 314, 1585-8.

(44) Choi, H. M. T.; Chang, J. Y.; Trinh, L. A.; Padilla, J. E.; Fraser, S. E.; Pierce, N. A. Nat. Biotechnol. 2010, 28, 1208-12.

(45) Venkataraman, S.; Dirks, R. M.; Rothemund, P. W. K.; Winfree, E.; Pierce, N. A. Nat. Nanotechnol. 2007, 2, 490-4.

(46) Zhang, D. Y.; Chen, S. X.; Yin, P. Nature Chem. 2012, 4, 20814.

(47) Dueber, J. E.; Wu, G. C.; Malmirchegini, G. R.; Moon, T. S.; Petzold, C. J.; Ullal, A. V; Prather, K. L. J.; Keasling, J. D. Nat. Biotechnol. 2009, 27, 753-9.

(48) Whitaker, W. R.; Davis, S. A; Arkin, A. P.; Dueber, J. E. Proc. Natl. Acad. Sci. U.S.A. 2012, 109, 18090-5. 